Research Article

\title{
Simulation for Sludge Flocculation I: Brownian Dynamic Simulation for Perikinetic Flocculation of Charged Particle
}

\author{
Linshuang Liu, ${ }^{1,2}$ Guolu Yang, ${ }^{1,2}$ and Minghui $\mathbf{Y u}^{2}$ \\ ${ }^{1}$ Sewage Sludge \& Silt Research Center, Wuhan University, Wuhan 430072, China \\ ${ }^{2}$ State Key Laboratory of Water Resource and Hydropower Engineering Sciences, Wuhan University, \\ Wuhan 430072, China \\ Correspondence should be addressed to Linshuang Liu, liu1965888@163.com
}

Received 27 October 2011; Revised 21 December 2011; Accepted 21 December 2011

Academic Editor: Zhijun Zhang

Copyright (C) 2012 Linshuang Liu et al. This is an open access article distributed under the Creative Commons Attribution License, which permits unrestricted use, distribution, and reproduction in any medium, provided the original work is properly cited.

\begin{abstract}
To investigate sludge drying process, a numerical simulation based on Brownian dynamic for the floc with uncharged and charged particles was conducted. The Langevin equation is used as dynamical equation for tracking each particle in a floc. An initial condition and periodic boundary condition which well conformed to reality is used for calculating the floc growth process. Each cell consists of 1000 primary particles with diameter $0.1 \sim 4 \mu \mathrm{m}$. Floc growth is related to the thermal force and the electrostatic force. The electrostatic force on a particle in the simulation cell is considered as the sum of electrostatic forces from other particles in the original cell and its replicate cells. It is assumed that flocs are charged with precharged primary particles in dispersion system by ionization. By the analysis of the simulation figures, on one hand, the effects of initial particle size and sludge density on floc smashing time, floc radius of gyration, and fractal dimension were discussed. On the other hand, the effects of ionization on floc smashing time and floc structure were presented. This study has important practical value in the high-turbidity water treatment, especially for sludge drying.
\end{abstract}

\section{Introduction}

There are numerous sediment-laden rivers in china. There are 42 rivers whose average annual sediment discharge is larger than 10 million. The sediment discharge of the Yellow River and the Yangtze River is $29.3 \%$ of the total top thirteen great rivers in the world. There are also many seasonal high-turbidity rivers on other regions of china. Therefore, the research of the high-turbidity water treatment has become a important research topic in china $[1,2]$.

The effect of moisture content on the sludge volume in enormous. Moisture of sludge which taken out from the lakes and rivers was very high. This led a lot of work needed to be 
done before sludge can be used as resources. The moisture associated with flocs of sludge is: $70-75 \%$ free water, $20-25 \%$ floc water, and $1 \%$ each capillary and bound water. The free water can be removed by thickening. The floc water is trapped in the interstices of floc particles and is separable only by mechanical drying [3]. However, as the sludge flocculation mechanism is complex, it is still difficult to dry vast amount of sludge rapidly. As a method of dehydration, flocculation has been often used in drying engineering. Therefore, flocculation mechanism and its dynamcis simulation are very important to improve the sludge drying speed in the future studies [4].

Sludge has many properties those differ from the coarse sediment and solution molecular. The floc of fine sediment particles occurs mostly in the flocculation process. In the sludge drying process, the particles grow to floc with electrochemical force [5] and adsorption [6]. These processes are very common in many hydrosol processes, especially for fine sediment flocculation. In the case of thermally stable particles, the Brownian motion should be considered as the dominant action. The aggregation characteristics are mainly affected by the morphology of aggregates [7]. As a fractal dimension can quantify the morphology of flocculation, it becomes a key variable for analyzing the flocculation dynamics. Ermak [8] simulated the flocculation process of charged particles in solution using Brownian dynamics. With more new applications for solid particles in solution recently, it is important to control the flocs size and its morphology in flocculation process. Xiong et al. [9] simulated the growth of game colloidal particle in the sectional colloid model. However, their results were obtained using the assumption of spherical particles. More work is necessary to better explain the realistic case. In this paper, we tried to simulate the motion of particles and analyse the effect of electrostatic force on the morphology in the sludge drying process. Therefore, we introduced a model that could describe the electrostatic force among charged sludge particles. This electrostatic simulation model was coupled with the Brownian dynamic simulation to deal with the flocculation of charged sludge particles. A comparison of floc growth process with different sludge density and particle diameters of charged and uncharged particles is present.

\section{Theory}

\subsection{Langevin Equation}

Brownian dynamics describe the dynamic behavior of a sediment particle whose mass and size are larger than those of the water molecules they are immersed in. These particles are subject to stochastic collisions with the water molecules, which leads to the random motion of the particles. It is assumed that a particle is in thermal equilibrium and surrounded by water molecules at a specific temperature $T$. The particles in this system are subject to an external force $R_{n}$. In this case, the translational motion is described by the Langevin equation. The strict Langevin equation for the motion of a particle on the $x, y, z$ coordinates is given by (2.1) together with several supplementary conditions $[10,11]$ :

$$
\begin{gathered}
\frac{d\left(m u_{n}\right)}{d t}=F_{n}-m \beta u_{n}+X_{n} \quad(n=x, y, z) \\
\left\langle X_{n}\right\rangle=0, \quad\left\langle X_{n}^{2}\right\rangle=2 \beta m k_{b} T, \quad\left\langle u_{n}(t) \cdot u_{n}(0)\right\rangle=0 \quad(n=x, y, z) .
\end{gathered}
$$

In (2.1), the left term of equation represents the Brownian particle's momentum, and the $R_{n}$ is systematic external force acting on the particle. In perikinetic flocculation, this term 
includes van der Waals force, electrostatic force, magnetic force, image force, and so forth. The other forces except for the electrostatic force can be negligible in the flocculation of charged sediment particles because the other forces are much smaller than the electrostatic force [5]. The specific calculation of the electrostatic force will be executed in Section 3. The second right term of equation is the frictional resistance of the fluid around the particle. $\beta$ is the friction constant, and it's the inverse of particle relaxation time. The last term $X_{n}$ is an external force caused by the random collisions between the particle and the surrounding water molecule. The random force $X_{n}$ is represented by the Gaussian random-distribution function. The mean and square mean of it are represented as (2.2).

\subsection{Solution of the Langevin Equation}

Several methods have been used to integrate the Langevin equation [12]. In this study, we use the Monte Carlo method. Integration of (2.1) (2.2) from $t$ to $t^{\prime}$ yields the equation:

$$
\begin{aligned}
& u_{n}-u_{0 n} e^{-\beta t}-\frac{F_{n 0}}{m \beta} \cdot\left(t-e^{-\beta t}\right)=\frac{1}{m} \int_{0}^{t} e^{-\beta\left(t-t^{\prime}\right)} X_{n}\left(t_{0}+t^{\prime}\right) d t^{\prime} \quad(n=x, y, z), \\
& r_{n}-r_{n 0} \frac{u_{n 0}}{\beta} \cdot\left(1-e^{-\beta t}\right)-\frac{F_{n 0}}{m \beta}\left[t-\frac{1}{\beta}\left(1-e^{-\beta t}\right)\right]=\frac{1}{m \beta} \int_{0}^{t}\left[1-e^{-\beta\left(t-t^{\prime}\right)}\right] X_{n}\left(t_{0}+t^{\prime}\right) d t^{\prime} .
\end{aligned}
$$

Equation (2.3) cannot be used directly to calculate the velocity and position changes due to the random nature of $X(t)$. We calculate the statistical properties of the two integrals involving $X(t)$ with one method derived by Luo et al. [11]

$$
\begin{aligned}
& u_{n}=u_{0 n} e^{-\beta t}+\frac{F_{0 n}}{m \beta} \cdot\left(t-e^{-\beta t}\right)+B_{1 n}, \\
& r_{n}=r_{n 0}+\frac{u_{n 0}}{\beta} \cdot\left(1-e^{-\beta t}\right)+\frac{F_{n 0}}{m \beta}\left[t-\frac{1}{\beta}\left(1-e^{-\beta t}\right)\right]+B_{2 n} \quad(n=x, y, z),
\end{aligned}
$$

where $B_{1 n}, B_{2 n}$ are random function of time chosen from the bivariate Gaussian distribution with properties

$$
\begin{aligned}
& \left\langle B_{1 n}\right\rangle=\left\langle B_{2 n}\right\rangle=0, \\
& \left\langle B_{1 n}^{2}\right\rangle=\frac{3 k T}{m}\left(1-e^{-2 \beta t}\right), \\
& \left\langle B_{2 n}^{2}\right\rangle=\frac{k T}{m \beta^{2}}\left(2 \beta t-3+4 e^{-\beta t}-e^{-2 \beta t}\right), \\
& \left\langle B_{1 n} \cdot B_{2 n}\right\rangle=0 \quad(i=1,2, \quad n=x, y, z) .
\end{aligned}
$$

\subsubsection{Floc Structure}

The morphological state of the flocs is defined as the fractal number by Forrest and Witten [13]. They confirmed that there was a power law relation between the characteristic length 
and the number of primary particles in each floc. Fractal structure of floc has been researched by many researches $[14,15]$. Generally, the radius of gyration of the flocculation is used as the characteristic length for the floc. The power law relation is expressed as

$$
R_{f}=\sqrt{\frac{1}{n} \sum_{1}^{n}\left(r_{j}-\frac{1}{n} \sum_{1}^{n} r_{j}\right)^{2}}
$$

where $n$ is the number of primary particles in an floc, $R_{f}$ is the radius of gyration.

\section{Numerical Simulation}

\subsection{Particle Charging}

When fine sediment dispersed in water, they were charged due to ionization and sorption $[16,17]$. In this simulation, we assumed that primary particles were charged with some additive ions to simulate the flocculation with different potential flocculent. The equilibrium and diffusive properties of charged spherical particles in solution have been studied by Xiangyan et al. [8], who simulated the motion of particles in solution obeying classical statistical mechanics. In this case, the charge quantity of a floc is the sum of elementary charges on the primary particles. The quantity of electricity on each particle is calculated by ionization intensity formula. In (3.1), $C_{i}$ is particle molarity, $I$ is ionization intensity, and $Z_{i}$ is quantivalency of particle. In our research, $C_{i}$ was relate to sludge density and the span of $I$ was $0.001 \sim 0.1$.

$$
I=\frac{1}{2} \cdot \sum_{1}^{n} C_{i} \cdot Z_{i}^{2}
$$

\subsection{Initial Condition}

To simulate the flocculation process, initial conditions for the position and the velocity of the primary particles have to be given in Figure 1 and Figure 2, respectively. The positions of these particles are obtained with a homogeneous distribution in 3 dimensions. It can be assumed that the primary particles experience only thermal force in the initial state. Therefore, we set the initial velocities with a normal random number generator that is characterized by the Gaussian distribution of zero mean and $k_{b} T / m_{0}$ variance.

The primary particles diameters were chosen from $0.1 \sim 4 \mu \mathrm{m}[18,19]$, and space between two particles $L_{0}$ were calculated with sludge density to coincided to real liquid dispersion. The space is decreased with the increase of sludge density $\rho_{s}$ as well as particle diameters $\rho_{p}$ as shown in Figure 3.

We calculated the trajectories of 1000 primary particles in a simulation cell. The cell size was determined by the sludge density $\rho_{s}$. Ten simulations for each initial condition were performed to obtain statistically meaningful average values. The particle diameter $d_{p}$ was value to $0.1,0.5,1.0,2.0$, and 4.0 um separately and $\rho_{s}$ was value to $1100,1075,1050,1025$, and $1010 \mathrm{~kg} \mathrm{~m}^{-3}$. In this work, we have fixed the values of $T$, particles density $\left(\rho_{p}\right)$ and $\Delta t$ to be $293 \mathrm{~K}, 2650 \mathrm{~kg} \mathrm{~m}^{-3}$, and $1 / 50 \mathrm{~s}$, respectively. These conditions were obtained from the experimental conditions in a flocculation process. 


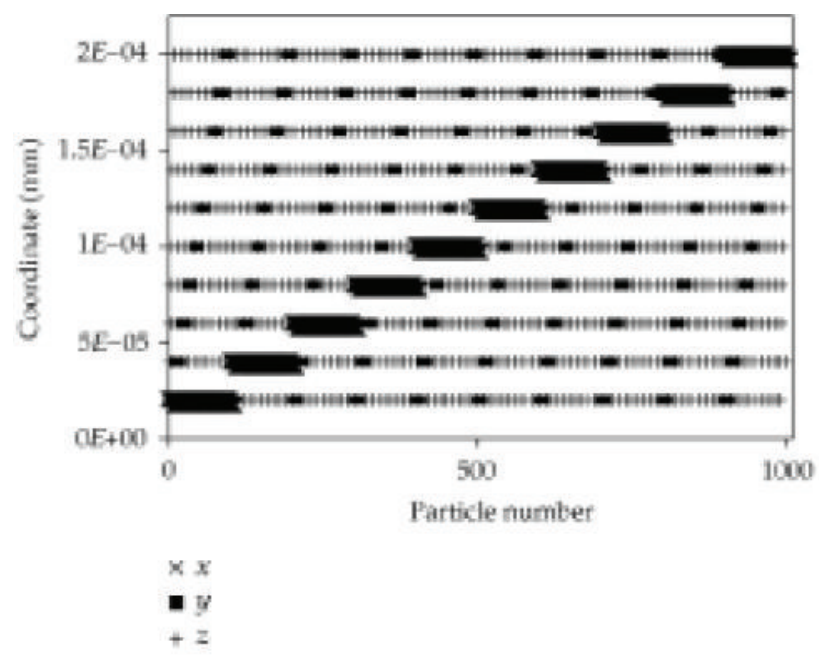

Figure 1: Particle initial position along $x, y, z$ axis.

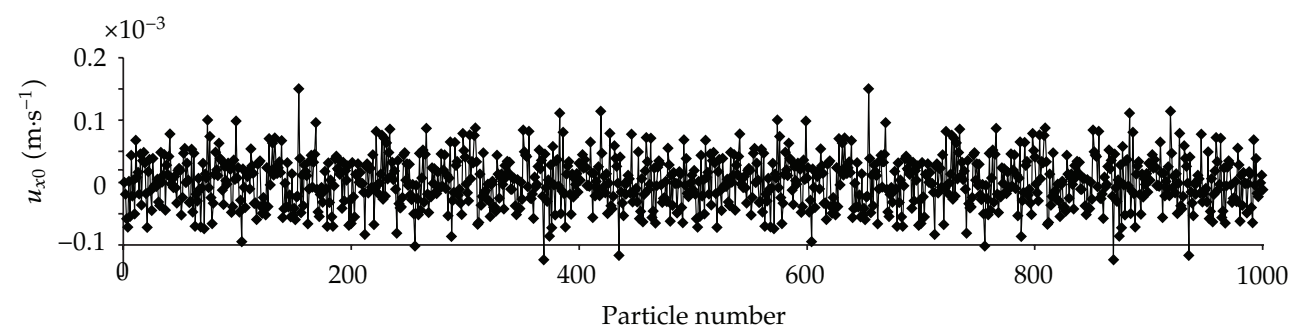

(a) $u_{x 0}$

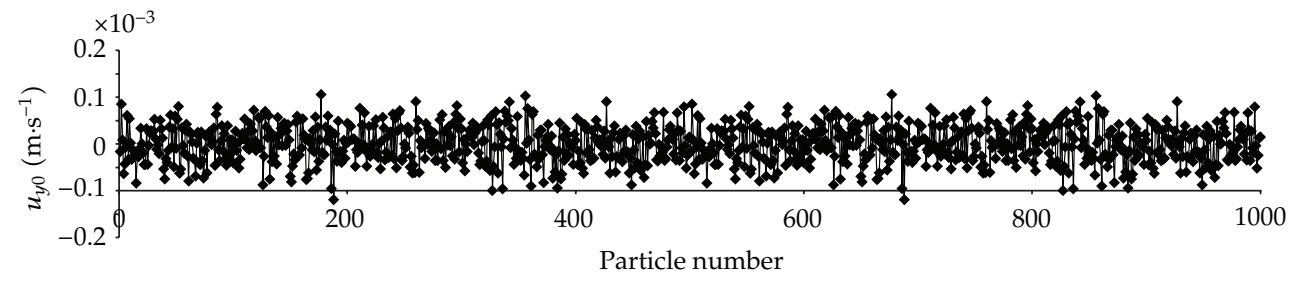

(b) $u_{y 0}$

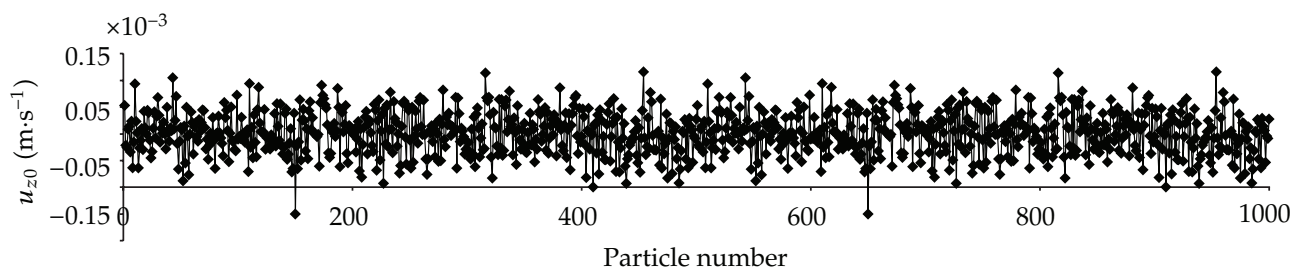

(c) $u_{z 0}$

Figure 2: Particle initial velocity along $x, y, z$ axis. 


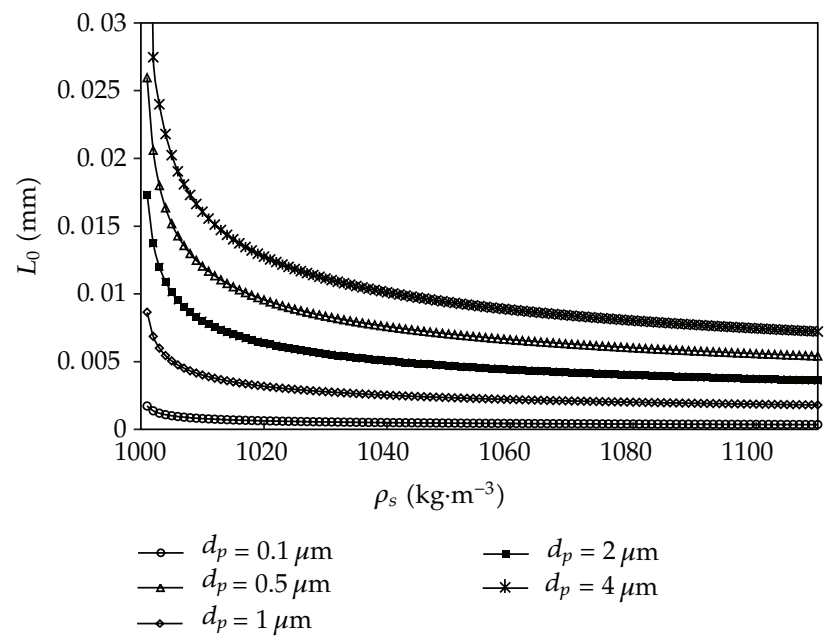

Figure 3: Space between Space between particles with different sludge density.

\subsubsection{Boundary Condition}

In this simulation, 300 particles were taken as boundary smash condition to signify the termination of floc growing. The periodic boundary condition was used not only for maintaining the internal bond strength but also for calculating the electrostatic force. The electrostatic force of the $i$ th particle is an infinite summation of each force from the other charged particles in the cell. Therefore, this force includes the electrostatic dispersion effect mentioned in the paper of Ermak [8]. The total electrostatic force on particle $i$ is given by

$$
F_{0 i}=\sum_{j=1, j \neq i}^{\infty} K_{e} \frac{q_{i} q_{j}}{r_{i j}^{2}} \overrightarrow{r_{i j}}
$$

In this paper, the electrostatic force can be calculated directly from the gradient of the pair potential. Ermak [8] introduced a new technique for treating an infinite number of pair potential. This potential includes the interaction with the images of the original cell in the replicated cells.

Though an infinite number of particles affect the electrostatic force of a given particle, the force has a finite value because it is symmetrical in a macroscopic sense. In our simulation, electric charges locate in the center of mass of the floc and the dielectric constant of the water is uniform. By applying this method to the present system, the modified electrostatic force by the infinite number of particles can be represented as:

$$
F_{0 i}=-\sum_{j=1, j \neq i}^{N} K_{e} q^{2}\left[\nabla \psi_{1}\left(r_{i j}\right)+\nabla \psi_{2}\left(\overrightarrow{r_{i j}}\right)\right],
$$

where $N$ is the total number of flocs in the original, cell; $q$ is quantity of charges on the particles. $\psi_{1}$ and $\psi_{2}$ are expressed as

$$
\psi_{1}(r)=\operatorname{erfc} \frac{(\sqrt{\pi} r / L)}{r}-\frac{1}{L}-\frac{E m}{L}
$$


Mathematical Problems in Engineering

$$
\psi_{2}(\vec{r})=\frac{1}{L} \sum_{\overrightarrow{1}}^{*}\left(\frac{\operatorname{erfc}(\sqrt{\pi}|(\vec{r} / L)+\vec{l}|)}{|(\vec{r} / L)+\vec{l}|}\right)+\frac{\exp \left(-\pi l^{2}\right) \cos (2 \pi \vec{l}(\vec{r} / L))}{\pi l^{2}},
$$

where $L$ is the length of the side of cell; Em is the Madelung constant and its value, for a simple cubic lattice, is $E m=2.83729479$ [20], $\overline{1}$ is a vector with integer components, $l$ is its magnitude, and $*$ denotes exclusion of the $\overline{1}=0$. To treat the equation of (3.5), we have used the optimized expansion in Cubic harmonics, which was first introduced by Xiangyan et al. [15]. Two basic matrix $P, Q$ in (3.7) are used in coefficient matrix $\left[k_{1}, k_{2}, k_{3}, k_{4}, k_{15}, k_{6}, k_{7}, k_{8}\right.$, $\left.k_{9}, k_{10}, k_{11}, k_{12}, k_{13}\right]^{T}$ to calculated the $\nabla \psi_{1}$ and $\nabla \psi_{2}$. In this method, 15 coefficients are listed in (3.8) by Hansen in order to optimize the (3.9)

$$
P=\left[\begin{array}{lll}
x_{1} & x_{2} & x_{3}
\end{array}\right] \quad Q=\left[\begin{array}{lll}
x_{1} & & \\
& x_{2} & \\
& & x_{3}
\end{array}\right]
$$

$$
\left[\begin{array}{l}
k_{1} \\
k_{2} \\
k_{3} \\
k_{4} \\
k_{5} \\
k_{6} \\
k_{7} \\
k_{8} \\
k_{9} \\
k_{10} \\
k_{11} \\
k_{12} \\
k_{13}
\end{array}\right]=\left[\begin{array}{c}
P Q \cdot(P Q)^{T} \\
P Q^{3} \cdot\left(P Q^{3}\right)^{T} \\
\exp \left(-\pi P \cdot P^{T}\right) \\
\left|Q^{2}\right| \\
\end{array}\right.
$$

$$
\left[\begin{array}{cccc}
a_{4} & b_{4} & c_{6} & \\
a_{6} & b_{6} & c_{8} & d_{8} \\
a_{8} & b_{8} & c_{10} & d_{10} \\
a_{10} & b_{10} & c_{12} & d_{12}
\end{array}\right]=\left[\begin{array}{cccc}
-3.090150 & 9.8010671 & -22.645201 & \\
4.6764131 & -19.994017 & -656.4341 & 357.74714 \\
97.681783 & -237.90423 & 440.65802 & -570.95501 \\
-110.95681 & 312.86629 & 96.932540 & 133.22453
\end{array}\right]
$$

Consequently, the gradients of $\nabla \psi_{1}$ and $\nabla \psi_{2}$ are represented as:

$$
\begin{gathered}
\nabla \psi_{1}\left(r_{i j}\right)_{/ n}=\frac{1}{L^{2}}\left(2 \frac{\exp \left(-\pi P \cdot P^{T}\right)}{P \cdot P^{T}}+\frac{\operatorname{erfc}\left(\sqrt{\pi P \cdot P^{T}}\right)}{\left(P \cdot P^{T}\right)^{3 / 2}}\right) x_{n} \cdot \widehat{e_{n}} \\
\nabla \psi_{2}\left(r_{i j}\right)_{/ n}=-\frac{1}{L^{2}}\left(m_{1} \frac{1}{x_{n}}+m_{2} x_{n}+m_{3} x_{n}^{3}+m_{4} x_{n}^{7}\right) \cdot \widehat{e_{n}} n=1,2,3,
\end{gathered}
$$


where $m_{1}, m_{2}, m_{3}, m_{4}$ are described as

$$
\begin{aligned}
m_{1}= & -\frac{2 k_{3} k_{11} k_{4}}{k_{5}^{2}} \\
m_{2}= & 2 \pi k_{3} k_{9}-k_{3} k_{6}+\frac{2 \pi k_{3} k_{8} k_{1}-k_{3} k_{7} k_{1}}{k_{5}^{2}}+\frac{4 \pi k_{3} k_{8} k_{1}+2 \pi k_{3} k_{11} k_{4}-k_{3} k_{10} k_{4}}{k_{5}^{3}} \\
& +\frac{6 k_{3} k_{11} k_{4}+2 \pi k_{3} k_{13} k_{2}-k_{3} k_{2} k_{12}}{k_{5}^{4}} \\
& +\frac{k_{2} k_{3} k_{13} k_{2}}{k_{5}^{5}}, \\
m_{3}= & -\frac{4 k_{3} k_{8}}{k_{5}^{2}} \\
m_{4}= & -\frac{8 k_{3} k_{13}}{k_{5}^{4}} .
\end{aligned}
$$

\subsection{Calculation Procedure}

We define that a particle collides with the floc if the distance between two is located within the radius of gyration of floc. The momentum and the charge quantity are conserved before and after the collision, and immediately the new-formed floc is in thermal equilibrium state. We assumed that the sticking probability was 1 after each collision. The flocs grow with the repetition of the above sequence. The program was written with Fortran 95 and the procedure is shown in Figure 4.

\section{Results}

\subsection{Floc Growth Process}

\subsubsection{Number of Particles in Floc}

A series of comparison of $d_{p}$ from 0.1 to 4.0 um were performed to interpret floc growth process. We chose $\rho_{s}=1010,1025,1050,1075$, and $1100 \mathrm{~kg} \mathrm{~m}^{-3}$ for simulation in order to intergrate with practice. As shown in Figure 5, in the early stage of flocculation the primary particle was sustaining to the a initial flocculent time. The particle number begun to increase after the first stage till reaching the smashing condition. In the final stage of flocculation, the sequence of smashing time with different sludge density is similar to that of initial flocculent time. The initial flocculent time and smashing time are both increased as the floc diameter increased.

\subsubsection{The Smashing Time}

The floc size can not grow up unlimited in the practice. It could be affect by particle diameter and particle-bonding mode. In this paper, the time that 300 particles conglutinated in one floc 


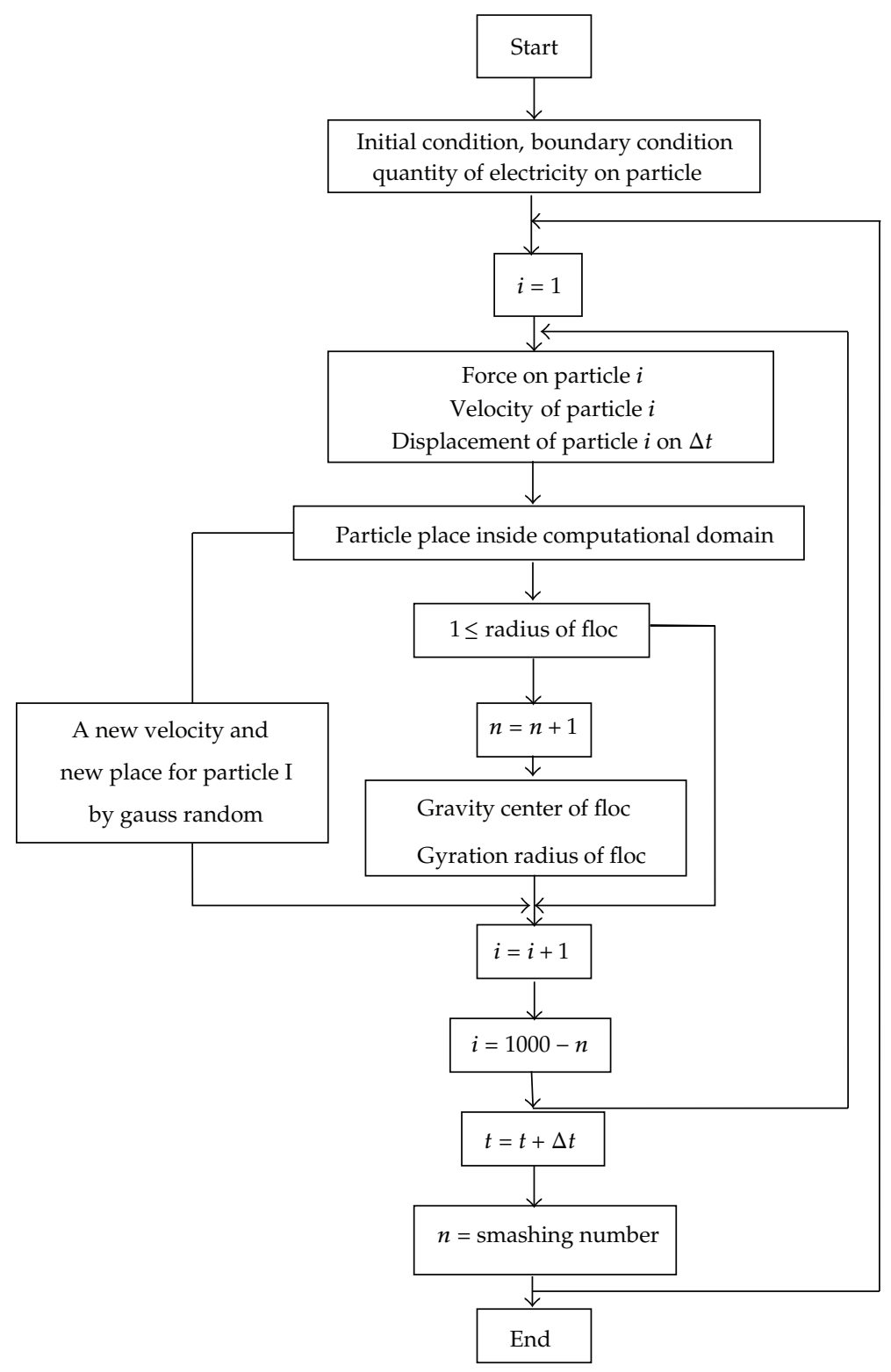

Figure 4: Flow chart for Brownian dynamic simulation.

is smashing time. Though smashing time was related to random velocity and displacement, it increased with the decrease of sludge density. Whereas it increased with the increase of particle diameter as shown in Figures 6 and 7. This tendency may be understood, on one hand as particle diameter presents a linear increase, the particle number decreased in a cube way, which leads particle impact probability to decrease. On the other hand, the decrease of sludge density causes particle impact probability to decrease likewise. 


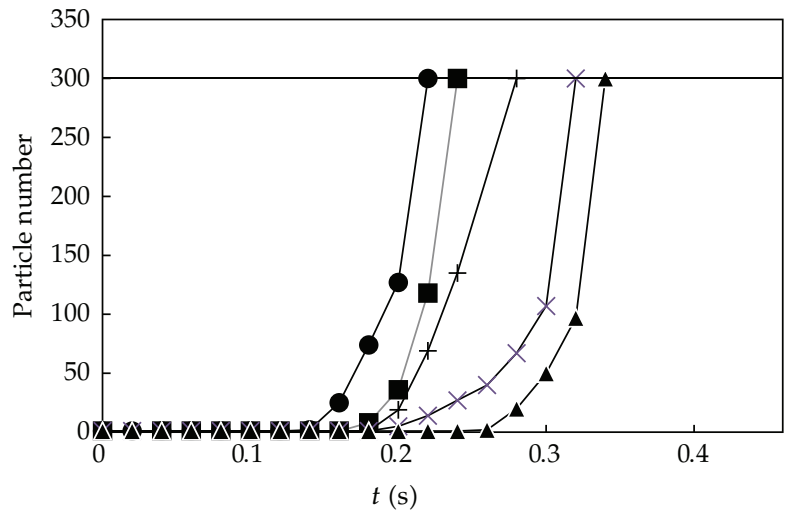

(a) $d p=0.1$

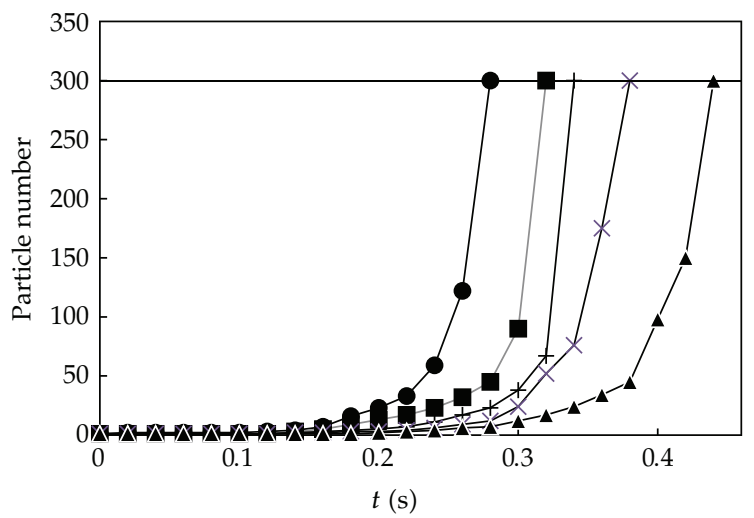

(b) $d p=1 u m$

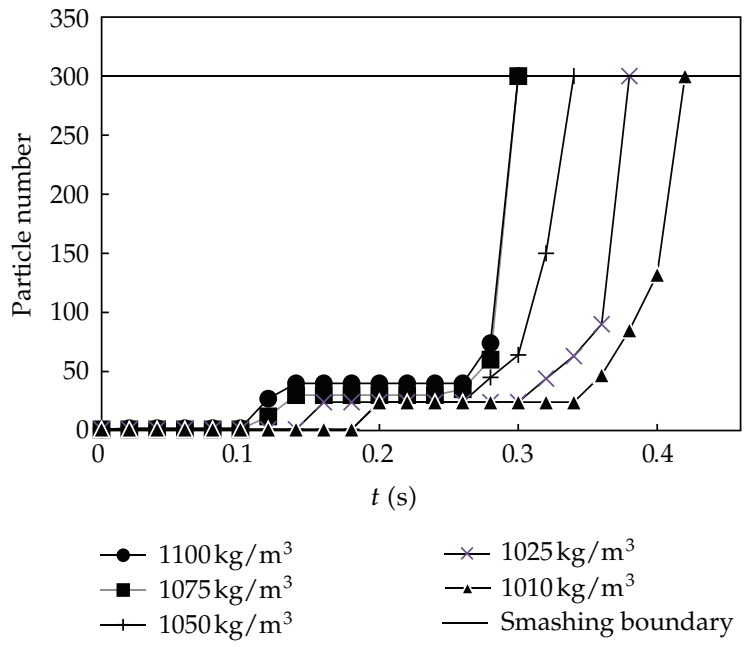

(c) $d p=4 u m$

Figure 5: Variety of particle number as floc growing. 


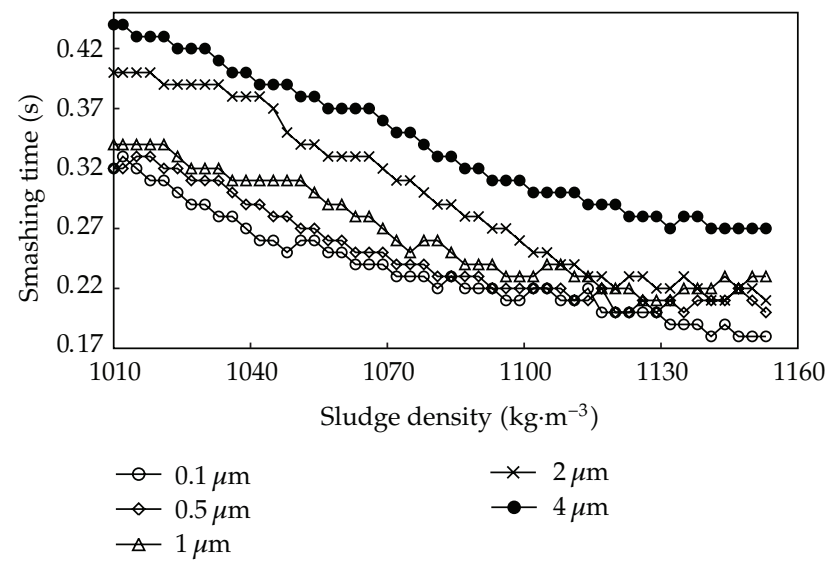

Figure 6: Effects of sludge density on smashing time.

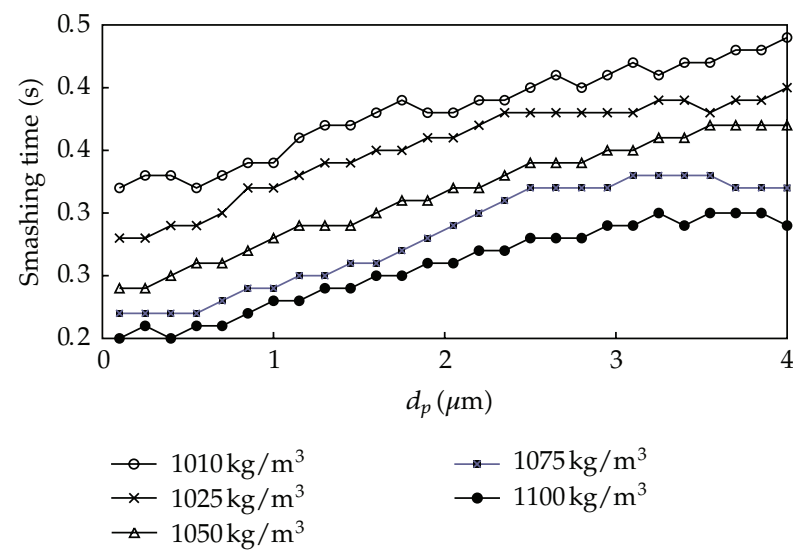

Figure 7: Effects of particle diameter on smashing time.

\subsubsection{Radius of Gyration}

The relation between the radius of gyration and particle number was not linear positive correlation. The main factors influencing it were particle number and fractal dimension. In this simulation, the radius of gyration is calculated by four variables, they were particle number and $x-, y-, z$-axis coordinate values. The radius of gyration values in Figure 8 was calculated by three duplicate computations with the initial particle size being equal to $2 \mathrm{um}$. It was shown in the Figure 8 that the gyration radius decreased with the increase of sludge density. The relation formulas of sludge density to gyration radius is $R_{f}=-1441\left(\rho_{s}\right)^{3}+4616\left(\rho_{s}\right)^{2}-$ $4927\left(\rho_{s}\right)+1752$, and correlation coefficient is 0.992 .

\subsubsection{Fractal Dimension}

The fractal dimension was calculated with gyration radius method. the mean values of fractal dimension of the three series were shown in Figure 9. The relation formulas of sludge density to gyration radius is $D_{f}=1.3338 \pm 0.0451+(0.6049 \pm 0.1424) \times\left(\rho_{s}-1000\right)^{0.4107 \pm 0.4181}$, and 


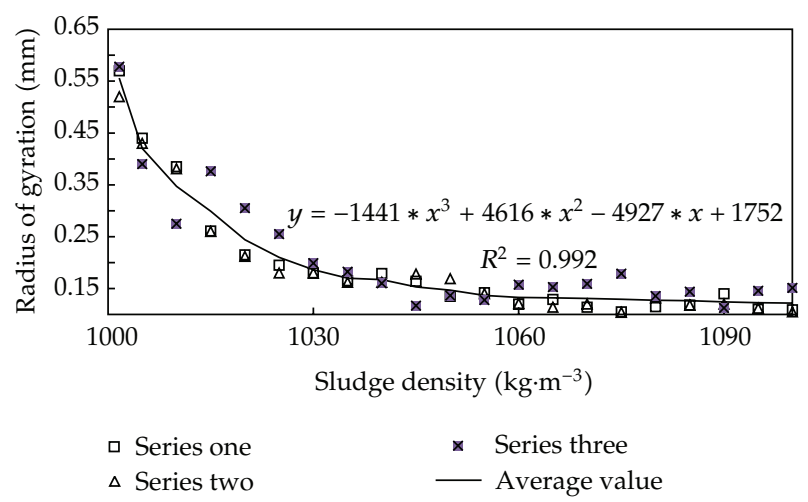

Figure 8: Effects of sludge density on floc diameter.

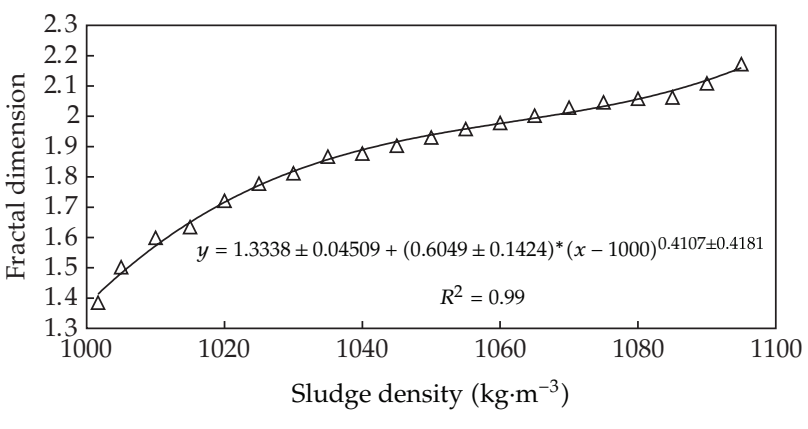

$\Delta$ Aaverage value

Figure 9: Effects of sludge density on fractal dimension.

correlation coefficient is 0.991 . The gyration radius is increased with the increase of sludge density. The reason is that with the increase of sediment density, the collision probability of sediment particles and floc increased, while the opportunities for floc to accept new particles increased, which finally made the floc more compact. Several authors $[21,22]$ have researched the effects of sludge density on floc fractal dimension with numerical simulation method. The results show that relation formula of sludge density to gyration radius with Diffusion Limited Cluster Aggregation model is $D_{f}=D_{f 0}+a \Phi^{c}$, which is similar to our conclusion. Xiangyan et al. [15] has studied the effect of sludge density on gyration radius in two-dimensional space and the results presented in their paper are also similar to our conclusion.

\subsection{Morphology of Floc}

The morphology of the floc is a very important factor to determine the dynamic behavior of flocs. The morphology of a floc is visualized for the case of uncharged particles in Figure 10(a). This floc, consisting of 300 primary particles, is obtained from a simulation based on 1000 primary particles with edge length decided by sludge density. Its structure is quite open to the outer space and it is very similar to the structures of aggregates observed by other researchers [21-23]. 


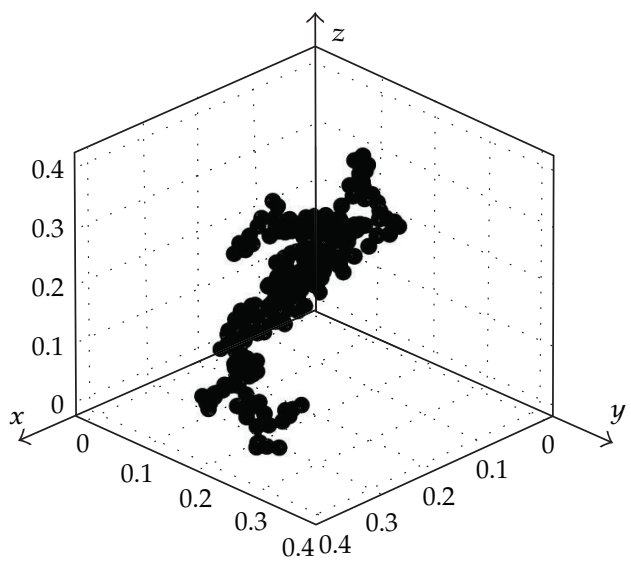

(a) Floc formed by uncharged particles

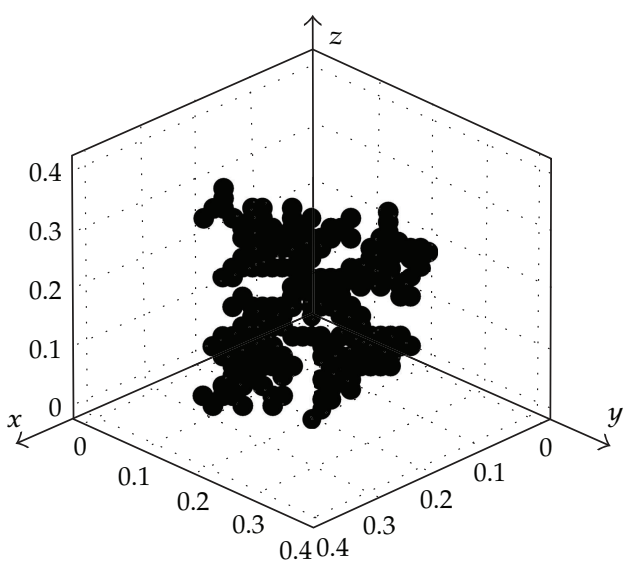

(b) Floc formed by charged particles, $q=-2.0 \times 10^{-14} \mathrm{C}$

Figure 10: Morphology of floc.

\subsection{Effects of Electrostatic Force on Flocs}

Figure 11 shows a comparison of the simple Coulomb force and the modified electrostatic force. The Coulomb force was calculated just within only one cell. There is no difference if two particles are close together, there is a relatively large difference as the distance becomes larger. Because of the periodic boundary condition, the electrostatic force must be zero if two particles are located at center and the edge of the simulation cell. The modified electrostatic force satisfies this condition well. Therefore, our model for the electrostatic force can be regarded as a good model coinciding with the real system. The morphology is quantified with a fractal dimension. It is affected by the number of primary particles and the radius of gyration, as shown in Figure 10(b). In this condition, the primary particles have $-2.0 \times 10^{-14} \mathrm{C}$ quantity of electricity according to (3.1).

Four serieses of growth process are simulated to present the effects of charges on smashing time. As shown in Figure 12, the effect of electric quantity on floc is obvious. However, the effects of unipolar and polar electrical charges on floc are still needed to be researched in the further work. Figures 13(a)-13(c) showed the effect of charges on smashing time. The smashing time is increased with sludge density as well as electrical charges.

\section{Conclusions}

To investigate sludge drying process, a numerical simulation for the floc of uncharged and charged particles was presented with a Brownian dynamic. The initial conditions for the particle position were contained by sludge density and velocity was given with Gaussian distribution. To treat the electrostatic force, a modified electrostatic force has been introduced.

In a fixed volume of water, the particle number increases gradually as the time and sludge density increase. In the meantime its increasing rate increases with time. However, it decreases as the increase of particle diameter. Though the smashing time was related to random velocity and displacement, it increased as the decrease of sludge density, yet it increased as the increase of initial particle diameter. 


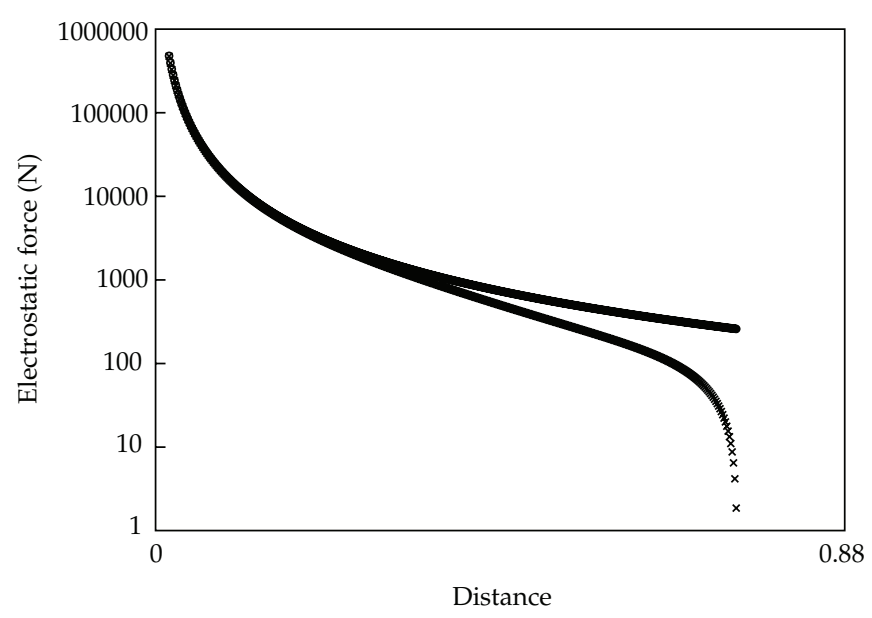

$\times$ Coulomb force

- Modified electrostatic force

Figure 11: Comparison between modified electrostatic force versus simple Coulomb force.

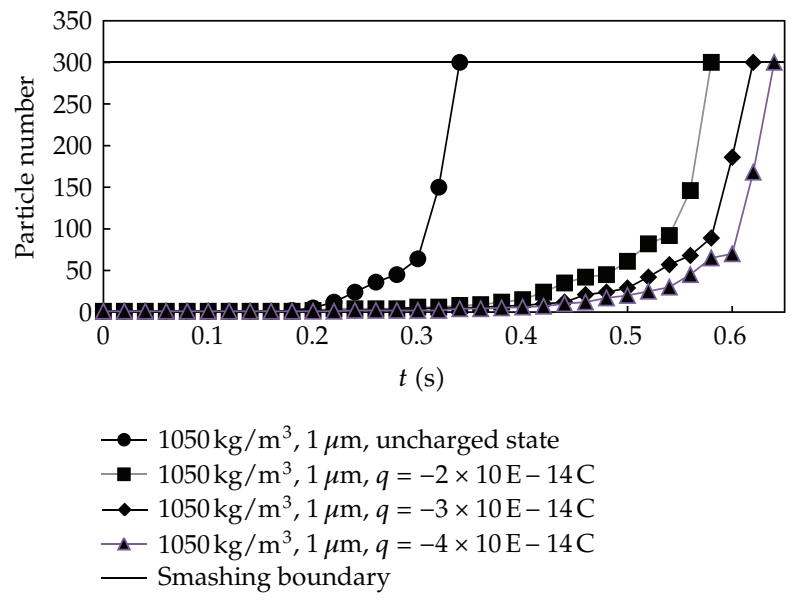

Figure 12: Variety of particle number as floc growing.

The gyration radius is increased with the increase of sludge density. The relation formula of sludge density to fractal dimension is $R_{f}=-1441\left(\rho_{s}\right)^{3}+4616\left(\rho_{s}\right)^{2}-4927\left(\rho_{s}\right)+1752$, and correlation coefficient $\left(R_{2}\right)$ is 0.991 . The fractal dimension was calculated with gyration radius method. The relation formulas of sludge density to gyration radius is is $D_{f}=1.3338 \pm$ $0.0451+(0.6049 \pm 0.1424) \times\left(\rho_{s}-1000\right)^{0.4107 \pm 0.4181}$, and correlation coefficient is 0.990 . The conclusion is similar to the results of previous studies.

Effects of electric quantity on floc were obvious. The smashing time was increased with the increase of electric quantity. Besides, the polar electrical charges on primal particles make the morphology of floc more open. However, this simulation was carried out under the assumption of the high-moisture content. Therefore, our conclusions cannot be applied directly to other cases, where the moisture content is extremely low. To find out the particles motiom 


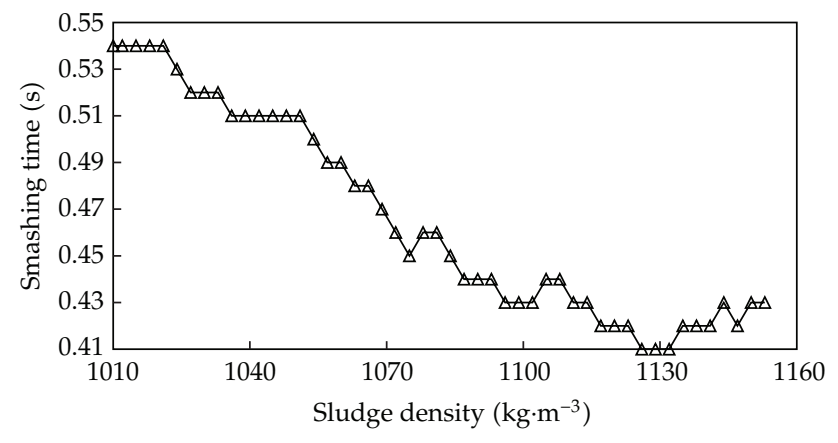

(a) $q=-2 \times 10^{-14} \mathrm{C}$

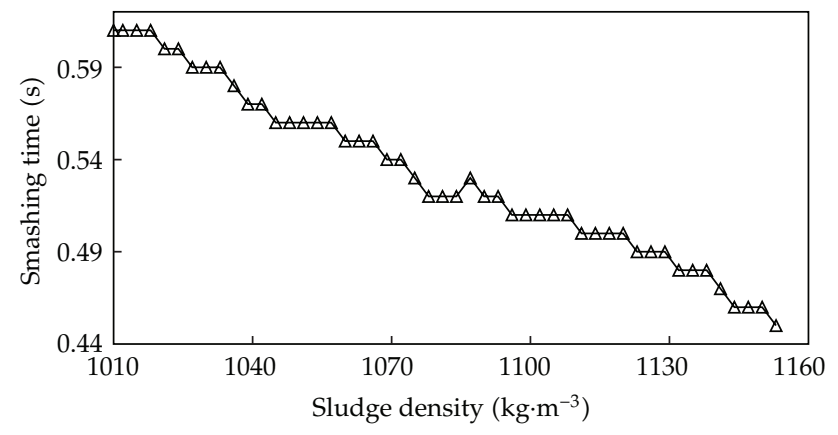

(b) $q=-3 \times 10^{-14} \mathrm{C}$

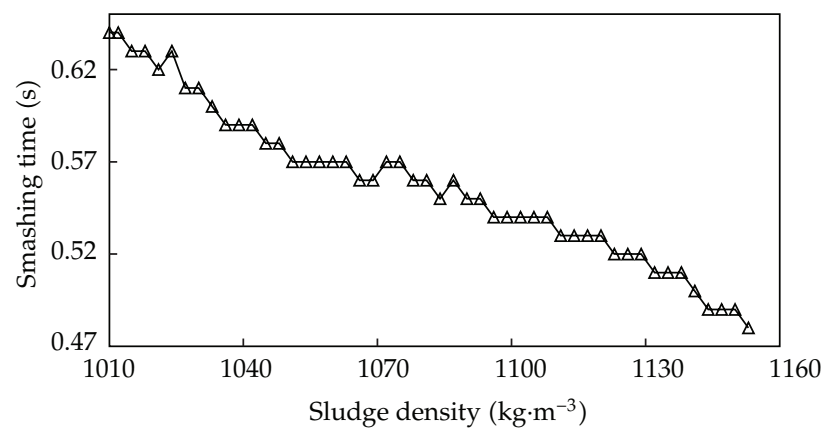

(c) $q=-4 \times 10^{-14} \mathrm{C}$

Figure 13: Effects of sludge density on smashing time.

law in the low moisture content, it needs some additional models, and we will perform additional simulations and experiments to confirm it as future works.

\section{Notation}

T: $\quad$ Disperse system temperature, $\mathrm{k}$

$t, t^{\prime}$ : Time, $\mathrm{s}$

$\Delta t$ : Time step, $\mathrm{s}$

$m$ : Mass of aggregate, $\mathrm{kg}$ 
$R_{n}$ : External force, $\mathrm{N}$

$u_{n}$ : Particle velocity, $\mathrm{m} / \mathrm{s}$

$X_{n}$ : External force caused by the random collisions, $\mathrm{N}$

F: Electrostatic force, $\mathrm{N}$

$q$ : Charge quantities on particle, $\mathrm{C}$

$r$ : Displacement of aggregate, $\mathrm{m}$

$L$ : Length of side of simulation cell, $m$

$L_{0}$ : Initial space between particles, $\mathrm{m}$

$d_{p}$ : Particle diameter, $\mathrm{m}$

$R_{f}$ : Radius of gyration for aggregate, $\mathrm{m}$

$D_{f}$ : Fractal dimension

$n$ : Total number of particle in one floc, dimensionless

$\beta$ : $\quad$ Friction constant, dimensionless. $\mathrm{S}^{-1}$

$\Phi: \quad$ Electrostatic potential energy, $\mathrm{kg} \cdot \mathrm{m}^{2}$

$\rho_{s}$ : Sludge density, $\mathrm{kg} \cdot \mathrm{m}^{-3}$

$\rho_{p}$ : Particle density, $\mathrm{kg} \cdot \mathrm{m}^{-3}$

I: Charge density, dimensionless

$C_{i}$ : Particle molar density, $\mathrm{mol} \cdot \mathrm{m}^{-3}$

$Z_{i}$ : The total valence of particle surface, dimensionless

$E_{p}$ : Madelung constant, dimensionless

$k_{b}$ : Boltzmann constant, dimensionless

$k_{e}$ : Coulomb constant, dimensionless.

\section{Acknowledgments}

The authors would like to thank Associate Professor Wu Weimin and Associate Professor Yu Liangying for helpful discussions. This study was supported by the National Key Basic Research Development (973) program of China (Grant no. 2011CB403300).

\section{References}

[1] J. Yajun, A Study on Characteristic and Mechanism of Spontaneous Sedimentation of Yellow River's Loess Oarticles, Xi'an University of Architecture and Technology, 2004.

[2] L. Ligang, The Study of the Rule of Sediment Movement and Deposit and Operation of Reducing Sediment for Xiaolangdi Reservoir of Yellow River, Hohai University, 2005.

[3] T. J. McGhee, Water Supply and Sewerage, New York, NY, USA, 1991.

[4] J. Werther and T. Ogada, "Sewage sludge combustion," Progress in Energy and Combustion Science, vol. 25, no. 1, pp. 55-116, 1999.

[5] H. Helmholtz, "Ueber galvanische Ströme, verursacht durch Concentration sunters chiede, Folgerungen aus der mechanischen Wärmetheorie," Viedemann' Annalen, no. 7, p. 337, 1879.

[6] S. Mishra, A. Mukul, G. Sen, and U. Jha, "Microwave assisted synthesis of polyacrylamide grafted starch (St-g-PAM) and its applicability as flocculant for water treatment," International Journal of Biological Macromolecules, vol. 48, no. 1, pp. 106-111, 2011.

[7] T. Matsoukas and S. K. Friedlander, "Dynamics of aerosol agglomerate formation," Journal of Colloid And Interface Science, vol. 146, no. 2, pp. 495-506, 1991. 
[8] D. L. Ermak, "A computer simulation of charged particles in solution. I. Technique and equilibrium properties," The Journal of Chemical Physics, vol. 62, no. 10, pp. 4189-4196, 1975.

[9] Y. Xiong, S. E. Pratsinis, and S. V. R. Mastrangelo, "The effect of ionic additives on aerosol coagulation," Journal of Colloid And Interface Science, vol. 153, no. 1, pp. 106-117, 1992.

[10] B. H. McNaughton, P. Kinnunen, M. Shlomi et al., "Experimental system for one-dimensional rotational Brownian motion," Journal of Physical Chemistry B, vol. 115, no. 18, pp. 5212-5218, 2011.

[11] J. P. Luo, Z. M. Lu, and Y. L. Liu, "Simulation of Lagrangian dispersion using a Lagrangian stochastic model and DNS in a turbulent channel flow," Journal of Hydrodynamics, vol. 21, no. 6, pp. 767-773, 2009.

[12] P. Turq, F. Lantelme, and H. L. Friedman, "Brownian dynamics: its application to ionic solutions," The Journal of Chemical Physics, vol. 66, no. 7, pp. 3039-3044, 1976.

[13] S. R. Forrest and T. A. Witten, “Long-range correlations in smoke-particle aggregates," Journal of Physics A, vol. 12, no. 5, pp. L109-L117, 1979.

[14] J. H. Jeon and R. Metzler, "Fractional Brownian motion and motion governed by the fractional Langevin equation in confined geometries," Physical Review E, vol. 81, no. 2, Article ID 021103, 2010.

[15] K. Xiangyan, L. I. Daolun, and X. U. Xianzhi, "Study on the mathematical models of coupled thermalhydrological -mechanical (THM) processes," Journal of Hydrodynamics, vol. 4, pp. 34-41, 2005.

[16] S. G. Brush, H. L. Sahlin, and E. Teller, "Monte carlo study of a one-component plasma. I," The Journal of Chemical Physics, vol. 45, no. 6, pp. 2102-2118, 1966.

[17] D. L. Ermak, "A computer simulation of charged particles in solution. I. Technique and equilibrium properties," The Journal of Chemical Physics, vol. 62, no. 10, pp. 4189-4196, 1975.

[18] P. N. Pusey, "Brownian motion goes ballistic," Science, vol. 332, no. 6031, pp. 802-803, 2011.

[19] A. Einstein, Investigations on the Theory of Brownian Movement, Edited by R. Furth, Dover, New York, NY, USA, 1956.

[20] R. Cherian, C. Gerard, P. Mahadevan, N. T. Cuong, and R. Maezono, "Size dependence of the bulk modulus of semiconductor nanocrystals from first-principles calculations," Physical Review B, vol. 82, no. 23, Article ID 235321, 7 pages, 2010.

[21] J. Pengkang, W. Xiaochang, and G. Kun, "Dla simulation of fractal flocs and calculation of fractal dimension," Environmental Chemistry, vol. 1, no. 26, pp. 5-9, 2007.

[22] F. A. Campo, J. S.R. Murillo, and E. J. Barbero, "Aggregation model for the gelation of a sol starting from the processing conditions," Journal of Non-Crystalline Solids, vol. 357, no. 10, pp. 2046-2053, 2011.

[23] Q. Fu and K. Q. Xia, "Wavelet analysis of multifractal and its application to processing temperature data in Rayleigh-Benard convection," Journal of Hydrodynamics, vol. 13, no. 4, pp. 34-41, 2001. 


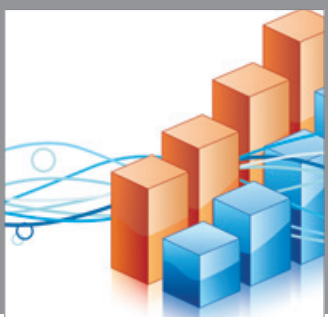

Advances in

Operations Research

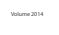

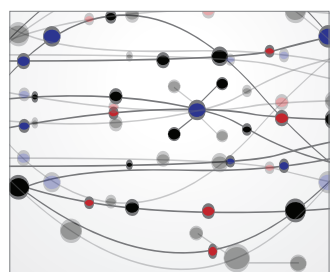

\section{The Scientific} World Journal
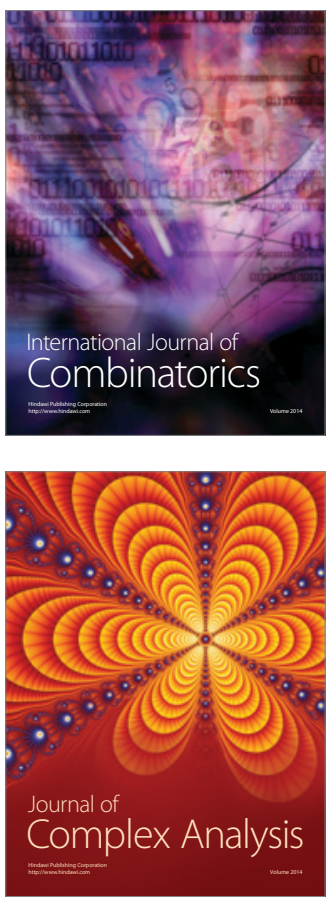

International Journal of

Mathematics and

Mathematical

Sciences
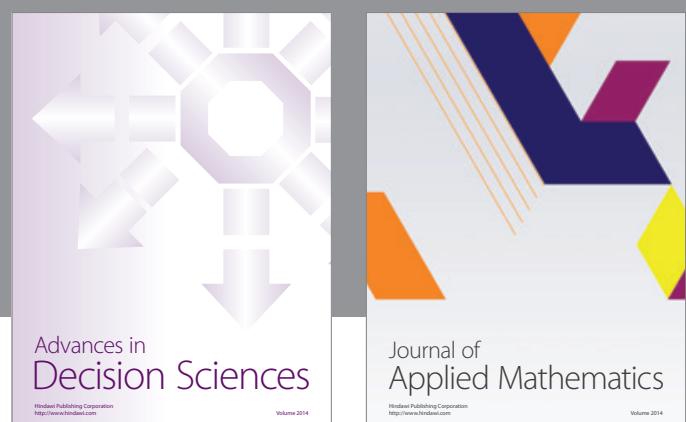

Journal of

Applied Mathematics
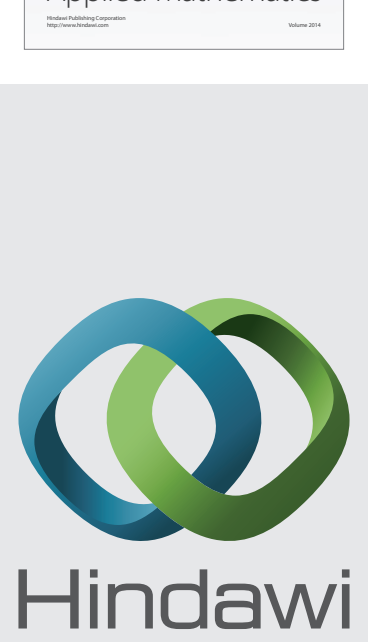

Submit your manuscripts at http://www.hindawi.com
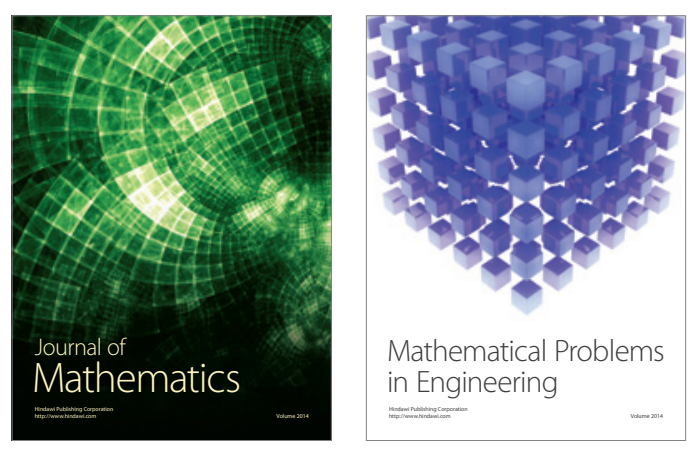

Mathematical Problems in Engineering
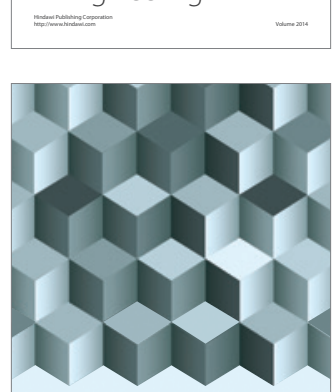

Journal of

Function Spaces
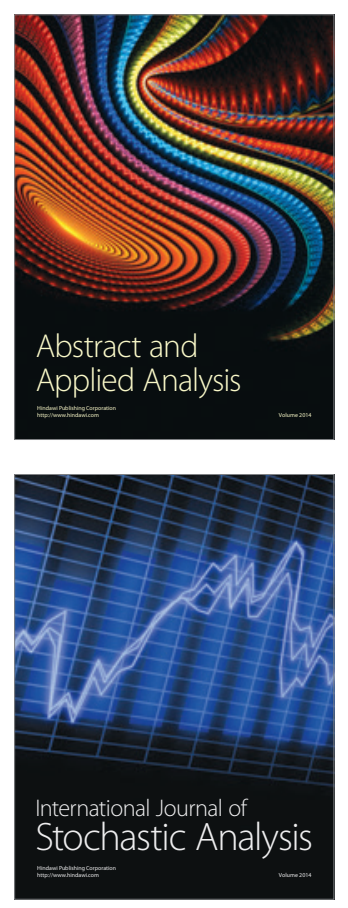

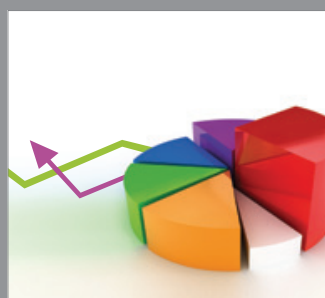

ournal of

Probability and Statistics

Promensencen
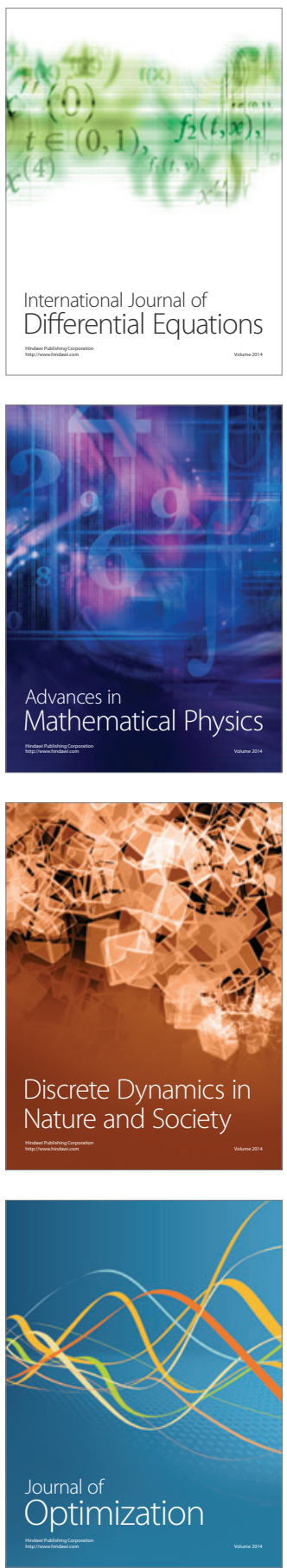